\title{
FINITE GROUPS CONTAINING MANY INVOLUTIONS
}

\author{
AVINOAM MANN \\ (Communicated by Ronald M. Solomon)
}

\begin{abstract}
If the ratio of the number of involutions of a finite group to the group order is bounded below, the group is bounded by abelian by bounded.
\end{abstract}

One of the first exercises in group theory is that a group in which all nonidentity elements have order two (so-called involutions) is abelian. An almost equally easy exercise states that a finite group is abelian if at least $3 / 4$ of its elements have order two. This cannot be improved, as the dihedral group of order eight, as well as its direct product with any elementary abelian group, provides examples of groups in which the number of involutions is exactly one less than 3/4 of the group order. However, it is known that groups in which many elements are involutions are not far from being abelian. Indeed, all groups in which at least half the elements are involutions have been determined by Miller and more recently by Wall and by Liebeck and MacHale (see [LM], which includes references to the earlier determinations); this was sharpened by Rusin, who determined the groups in which at least $11 / 32$ of the elements are involutions $[R]$. The following result formalises the sense in which the existence of many involutions makes a group nearly abelian.

Theorem. Let $G$ be a finite group of order $|G|$, and assume that at least $r|G|$ elements of $G$ are involutions, for some real number $r$. Then $G$ contains a normal subgroup $H$ such that both $|G: H|$ and $\left|H^{\prime}\right|$ are bounded by some function of $r$.

As will be seen, this follows easily by combining a formula of Frobenius and Schur with a recent result of Neumann. Our motivation, however, was an even more recent paper of Berkovich [Be] (see also [NB] and [BM]) which discusses the function $T(G)$ defined below, and I wish to thank this author for communications of this and other papers prior to publication and fruitful contacts generally.

If $G$ is a finite group, we denote by $k=k(G)$ the number of conjugacy classes of $G$, let $d_{1}, \ldots, d_{k}$ be the degrees of the irreducible characters of $G$,

Received by the editors January 20, 1993.

1991 Mathematics Subject Classification. Primary 20B05, 20D60.

Key words and phrases. Involutions. 
and write $T(G)$ for the sum of these degrees. We thus have

$$
\begin{aligned}
T(G) & =\sum d_{i}, \\
|G| & =\sum d_{1}^{2} .
\end{aligned}
$$

Lemma 1. With this notation, we have

$$
\sqrt{|G|} \leq T(G) \leq \sqrt{|G| k}
$$

Proof. The left-hand inequality is immediate from (2), and the right-hand one follows from (1) and the Cauchy-Schwarz inequality, applied to the vectors $\left(d_{1}, \ldots, d_{k}\right)$ and $(1, \ldots, 1)$. (The referee pointed out that a similar argument occurs in [I], e.g., in the proof of Theorem 4.11. Moreover, after writing the first version of this note $I$ have learned that an inequality equivalent to the righthand side of (3) appears already in [NB, Lemma 2.3], proved by a different method.)

Lemma 2 (Frobenius-Schur). Let $t(G)$ be the number of elements of $G$ whose square is 1 (i.e., involutions and the identity). Then there exist numbers $\nu_{i}=$ 1,0 , or -1, for $i=1, \ldots, k$, such that

$$
t(G)=\sum \nu_{i} d_{i}
$$

See [I, Corollary 4.6]. An immediate corollary is

$$
t(G) \leq T(G) \text {. }
$$

Lemma 3. If $G$ is a finite group such that $k(G) \geq s|G|$, for some real number $s$, then $G$ contains a normal subgroup $H$ such that both $|G: H|$ and $\left|H^{\prime}\right|$ are bounded by some function of $s$.

This is Theorem 1 of [PMN]. Now assuming the hypothesis of the theorem, inequalities (5) and (3) show that $k(G) \geq r^{2}|G|$, and Lemma 3 finishes the proof.

Of course, there can be no converse to the theorem as stated. But the conclusion of the theorem is valid also under the assumption that $T(G) \geq r|G|$. Conversely, suppose that $G$ contains a subgroup $H$ such that $|G: H| \leq C$ and $\left|H^{\prime}\right| \leq C$, for some real number $C$. Since each irreducible character of $H$ is a component of one of $G$, we get $T(G) \geq T(H)$; looking only at linear characters yields $T(H) \geq\left|H: H^{\prime}\right|$. Thus, $T(G) \geq\left(1 / C^{2}\right)|G|$. We can thus say that the ratio $T(G) /|G|$ is bounded below if and only if $G$ is bounded by abelian by bounded. We thus add to the many interesting characterisations of the class of bounded by abelian by bounded groups. The earliest such characterisation, due to B. H. Neumann, is as groups covered by bounded permutable subsets [BHN]. Recent characterisations are Lemma 3 and the "permutability" results of [CLMR] and [Bl]. They can also be characterised by their group rings satisfying generalised polynomial identities ([P, 5.3.15]; I am grateful to A. Shalev for pointing this out to me).

ADDED IN PROOF

The inequality $t(G) \leq \sqrt{|G| k}$ already occurs in the Brauer-Fowler paper (Ann. of Math. (2) 62 (1955), 565-583). 


\section{REFERENCES}

[Be] Y. Berkovich, Counterexamples to a conjecture about the sum of degrees of irreducible characters, preprint.

[BHN] B. H. Neumann, Groups covered by permutable subsets, J. London Math. Soc. (2) 29 (1954), 236-248.

[BI] R. D. Blyth, Rewriting products of group elements. I, J. Algebra 116 (1988), 506-521.

[BM] Y. Berkovich and A. Mann, On sums of degrees of the irreducible characters of a finite group and its subgroups (in preparation).

[CLMR] M. Curzio, P. Longobardi, M. Maj, and D. J. S. Robinson, A permutational property of groups, Arch. Math. 44 (1985), 385-389.

[I] I. M. Isaacs, Character theory of finite groups, Academic Press, San Diego, 1976.

[LM] H. Liebeck and D. MacHale, Groups with automorphisms inverting most elements, Math. Z. 124 (1972), 51-63.

[NB] K. G. Nekrasov and Ya. G. Berkovich, Finite groups with large sums of degrees of irreducible characters, Publ. Math. Debrecen 33 (1986), 333-354. (Russian)

[P] D. Passman, The algebraic structure of group rings, Wiley-Interscience, New York, 1977.

[PMN] P. M. Neumann, Two combinatorial problems in group theory, Bull. London Math. Soc. 21 (1989), 456-458.

[R] D. J. Rusin, What is the probability that two elements of a finite group commute, Pacific J. Math. 82 (1979), 237-247.

Einstein Institute of Mathematics, Hebrew University, Givat Ram, Jerusalem 91904, ISRAEL

E-mail address: mannerms.huji.ac.il 